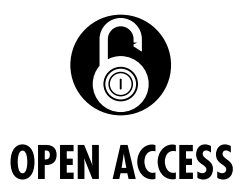

- Additional material is published online only. To view please visit the journal online (http://dx.doi.org/10.1136/ oemed-2018-105151).

${ }^{1}$ National Research Centre for the Working Environment, Copenhagen, Denmark ${ }^{2}$ Department of Biostatistics, University of Copenhagen, Copenhagen, Denmark ${ }^{3}$ Department of Occupational and Environmental Medicine, Bispebjerg University Hospital, Copenhagen, Denmark ${ }^{4}$ Department of Psychology, University of Copenhagen, Copenhagen, Denmark ${ }^{5}$ Department of Public Health, University of Copenhagen, Copenhagen, Denmark

Correspondence to Ida E H Madsen, National Research Centre for the Working Environment, Copenhagen DK-

Received 23 March 2018 Revised 29 June 2018 Accepted 9 July 2018 Published Online First 25 July 2018

\title{
Physical work demands and psychosocial working conditions as predictors of musculoskeletal pain: a cohort study comparing self-reported and job exposure matrix measurements
}

\author{
Ida E H Madsen, ${ }^{1}$ Nidhi Gupta, ${ }^{1}$ Esben Budtz-Jørgensen, ${ }^{2}$ Jens Peter Bonde, ${ }_{1}^{3}$ \\ Elisabeth Framke, ${ }^{1}$ Esben Meulengracht Flachs, ${ }^{3}$ Sesilje Bondo Petersen, ${ }^{3}$ \\ Annemette Coop Svane-Petersen, ${ }^{1}$ Andreas Holtermann, ${ }^{1}$ Reiner Rugulies ${ }^{1,4,5}$
} 2100, Denmark; ihm@nrcwe.dk

\begin{abstract}
Objectives Determining exposure to occupational factors by workers' job titles is extensively used in epidemiological research. However, the correspondence of findings regarding associations to health between job exposure matrices (JEMs) and individual-level exposure data is largely unknown. We set out to examine the prospective associations of physical work demands and psychosocial working conditions with musculoskeletal pain, comparing JEMs with individual-level self-reported exposures.
\end{abstract}

Methods We analysed data of 8132 participants from the Work Environment and Health in Denmark cohort study. Using random intercept multilevel modelling, we constructed age-specific and sex-specific JEMs estimating predicted exposures in job groups. We analysed associations between working conditions (individual and JEM level) at baseline and musculoskeletal pain at follow-up using multilevel modelling stratified by sex, adjusting for age, education and baseline pain.

Results Any consistent associations present in the individual-level analysis were also found in the JEM-level analysis. Higher pain levels at follow-up was seen for employees with higher baseline physical work demands, women exposed to violence and men with lower decision authority, whether measured at the individual or JEM level. Higher JEM-level quantitative demands were associated with less pain, but no association was seen at the individual level.

Conclusions We found predominately comparable prospective associations between working conditions and pain, whether using JEMs or individual level exposures, with the exception of quantitative demands. The results suggest that, with few notable exceptions, findings obtained using JEMs may be comparable with those obtained when using self-reported exposures.

Check for updates

(C) Author(s) (or their employer(s)) 2018. Re-use permitted under CC BY-NC. No commercial re-use. See rights and permissions. Published by BMJ.

To cite: Madsen IEH Gupta N, Budtz-Jørgensen E, et al. Occup Environ Med 2018:75:752-758

\section{INTRODUCTION}

Job exposure matrices (JEMs), for the assessment of working conditions, have long been applied in occupational medicine, in particular to assess chemical exposures (see, eg, refs 1 2). Regarding physical and psychosocial working conditions, JEMs are also not a new phenomenon, but there seems to be a growing interest in constructing

\section{Key messages}

What is already known about this subject? Job exposure matrices (JEMs) for physical work demands and psychosocial working conditions have been developed previously, but little is known regarding the correspondence between results obtained when analysing associations to health outcomes measuring working conditions using JEMs compared with individual-level measures. The few existing studies have analysed data crosssectionally.

\section{What are the new findings?}

This study is one of the first to report the correspondence between JEM-level measurements and individual-level measurements of physical work demands and psychosocial working conditions when analysed longitudinally in relation to changes in musculoskeletal pain. With a few exceptions, the associations were generally consistent whether applying individual or JEM level measurements.

\section{How might this impact on policy or clinical} practice in the foreseeable future?

The results qualify the examination of associations of physical work demands and psychosocial working conditions with risk of musculoskeletal disorders in large register-based studies that will provide important insights for policy makers and clinicians.

JEMs for these exposures. In recent years, several studies have applied or validated matrices based on self-reported exposure data aggregated to the job group level in Finland, ${ }^{3}{ }^{4}$ France, ${ }^{5}$ Australia ${ }^{6}$ and Denmark. ${ }^{78}$ In these JEMs, a group exposure estimate is constructed, representing the average assessment of the working conditions within the job group. This estimate can then be assigned to other populations without available exposure data, such as large-scale register-based cohorts with information on job titles and health-related information. Furthermore, when examining outcomes that cannot readily be measured without self-report, 
such as pain, the use of JEMs provide a possibility to circumvent reporting bias, which may be problematic when both exposure and outcome are self-reported. ${ }^{9}$ However, little is known, regarding whether the longitudinal association of working conditions with health outcomes are similar or different, when using JEM-based measurements compared with individual-level measurements. Five studies ${ }^{3-6}$ have reported associations of JEM and individual level psychosocial or physical working conditions with pain, mental health or self-rated health. However, these have been cross-sectional in nature, and there is a lack of evidence regarding longitudinal associations.

In this study, we examine the prospective associations of physical and psychosocial working conditions with musculoskeletal pain, and compare these associations when exposures are measured using a JEM approach and individual-level exposure data. To compare identical data at the JEM and individual level, we developed a sex-specific and age-specific JEM specifically for this study. We chose musculoskeletal pain as the outcome, because musculoskeletal disorders are leading causes disability, ${ }^{10}$ and it is an outcome, where previous findings regarding the role of the working conditions are mixed, ${ }^{11-16}$ which has been attributed, among other reasons, to varying types of exposure measurements. ${ }^{16}$

\section{METHODS}

We used data from The Work Environment and Health in Denmark cohort study (WEHD). WEHD was initiated to document developments in work environment and health in the Danish working population and based on a random sample of employed individuals aged 18-64 years. Data contain biannual questionnaire-based measures of self-reported working conditions from 2012 to 2020 . The present analyses used data from 2012 and 2014. There were 17662 respondents from the random sample of WEHD in 2012 (50\% response rate), of which 15872 were employed and 15198 could be grouped to a three-digit job group. Of these, 10891 responded at follow-up in 2014. After deleting participants with missing data on pain at baseline $(n=1446)$ or follow-up ( $n=1111)$, or any of the examined working conditions $(n=202)$, the final sample contained 8132 participants.

\section{Measurements}

Musculoskeletal pain

We measured musculoskeletal pain using a single-item modified version of the Nordic questionnaire for musculoskeletal symptoms, ${ }^{17}$ where respondents indicate if they during the past 3 months had pain in the following regions of the body: 'hips', 'knees', 'arms and/or wrists', 'neck and or/shoulders' and 'lower back', replying 'yes' or 'no' to each body region. All responses were summed to yield a score of the number of body regions with pain, ranging from 0 to 5 .

\section{Physical and psychosocial working conditions \\ Self-reported individual-level working conditions}

We included self-reported data from WEHD regarding the following physical work demands (supplementary appendix table A1): sitting, walking or standing, working with the back twisted or bent, arms lifted above the shoulders, repetitive arm movements, squatting kneeling, pushing or pulling and carrying or lifting. In line with previous research, ${ }^{18}$ we constructed a summary index for physical work demands by scoring each item from 1 to 6 (higher scores indicate higher demands, sitting reversely coded) and adding the scores. The index ranged from
8 to 48 . We also included data on the following psychosocial working conditions (supplementary appendix table A1): quantitative and emotional demands, decision authority, job insecurity and work-related violence. We included these exposures because previous research suggests that they are closely linked with job group and thus might be suitable for JEM estimation. ${ }^{819}$ We constructed scales ranging 1-5 for each exposure, except work-related violence and job insecurity (that were dichotomised), by scoring each item 1-5 and calculating the mean score.

We also included dichotomously defined variables for high exposure to each continuously measured working condition. We conducted this dichotomisation to allow for the possibility that the JEM might better identify the proportion of highly exposed individuals within job groups, than distinguish average differences in exposure levels. For physical, quantitative and emotional demands, and decision authority, we based the dichotomisation on the distribution of respondents, classifying approximately $10 \%$ of respondents as highly exposed. Job strain was defined as the combination of high quantitative demands and low decision authority, as defined by a median split of the continuous scores for these dimensions, in accordance with previous research. ${ }^{20} \mathrm{We}$ dichotomised the single item for job insecurity into high ('To a very high extent'/'To a high extent') or low ('To some extent'/“To a low extent'/(To a very low extent') and work-related violence into yes or no.

\section{Construction of the JEMs}

Based on the self-reported exposure data, we constructed JEMs for each exposure. Participants were categorised in job groups according to their DISCO-08 code, a Danish version of the International Standard Classification of Occupations (ISCO) developed by the International Labour Organization. ${ }^{21}$ The DISCO-08 codes were obtained through national Danish registers using monthly assigned job groups from Statistics Denmark's e-income Register. ${ }^{22}$ If no job group could be obtained from this register during 3 months before or after survey response, the primary job group during the year was used from the Employment Classification Module. ${ }^{22}$

In line with previous research, ${ }^{4}$ we required a minimum of 10 participants in each job group. If there were fewer than 10 participants in a group, this group was collapsed with similar small groups, and the participants were reclassified at a higher level of the DISCO code. As an example, participants belonging to groups ' 111100 Legislators', '111200 Senior Government Officials' and ' 111400 Senior Officials of Special-interest Organizations' were grouped together at the minor group level ' 111 Legislators and senior officials'. Participants who could not be grouped in this manner at the three-digit DISCO level or had no job group data were excluded from the analyses $(n=674)$. We used this strategy to avoid basing the JEM on groups with a small number of participants, yielding imprecise exposure estimates and conflating self-reported individual-level measurement with the exposure matrix.

To obtain JEMs that were sex-specific and age-specific, we used statistical modelling to construct the JEMs. For continuous variables, we estimated the predicted mean level of exposure using a generalised linear mixed model with random intercept for job group and the estimated best linear unbiased predictors. We constructed the matrices for men and women separately and inserted a fixed effect of age as a piecewise linear spline, with knots at the quartiles of the age distribution. For dichotomous variables, we estimated the predicted probabilities of exposure given job group, sex and age using a logistic 
model. All exposure matrices were constructed using SAS proc glimmix.

\section{Performance of the JEMs}

To assess the performance of the JEMs, we calculated intraclass correlation coefficients (ICCs) for continuous variables and area under the curve (AUCs) for dichotomous variables. The ICCs estimate the proportion of the total variance in exposure attributable to job group. The AUCs are known from receiver operating characteristics (ROC) curve analysis ${ }^{23}$ and measure the accuracy of a test by summarising the ROC curve. The AUC may be interpreted as the average level of sensitivity (in this case, the proportion of truly exposed classified as exposed by the test) of a measure across all possible levels of specificity (the proportion of truly non-exposed classified as non-exposed by the test). ${ }^{24}$ AUC levels may range from 0.50 (test is no better than chance) to 1 (perfect agreement). One suggestion for interpreting the AUCs has been to regard values of $\geq 0.70$ as fair, $\geq 0.80$ as good and $\geq 0.90$ as excellent. ${ }^{25}$ However, there is no gold standard for the interpretation of AUCs, and specific cut-off points are arbitrary and cannot be established in a scientifically sound manner. ${ }^{26}$ The AUCs of well-established screening tests may provide some context for interpretation. These include AUCs of 0.74-0.95 for mammography screening for breast cancer and 0.82 for MRI as a screening instrument for multiple sclerosis. ${ }^{26}$

The AUCs were estimated by comparing the JEMs developed on WEHD 2012 data to self-reported exposure data from WEHD 2014 in an independent sample of participants randomly drawn for WEHD 2014, linked using the 2014 job groups. We did not calculate AUCs cross-sectionally within the 2012 sample, because the self-reported information from this wave was used to construct the JEM and such analysis could overestimate the correspondence between self-reported data and JEM measurements as the two measures are not independent.

\section{Potential confounders}

As potential confounders, we included register-based data from year 2012 on sex and age from The Danish Civil Registration System ${ }^{27}$ and highest level of completed education from The Population's Education Register. ${ }^{28}$

\section{Statistical analyses of associations between working conditions and pain}

We analysed data using linear multilevel models with pain as a continuous outcome, including a random intercept across job groups to account for clustering of the JEM data at the job group level. We estimated the level of musculoskeletal pain at follow-up as a function of baseline working conditions, stratified by sex. We applied stepwise adjustment, with a minimally adjusted model including only baseline musculoskeletal pain and further models also including age and education.

\section{Sensitivity analyses}

As a sensitivity analysis, we repeated our analyses stratified by pain at baseline, dividing respondents into those with no pain at baseline and with pain at one or more locations at baseline. As results of the main analysis were similar for continuous and dichotomised versions of working conditions variables, this analysis was conducted only using the continuous measures where applicable. We additionally explored whether associations between physical work demands and pain were distinctive for different types of physical work demands by analysing scales for sitting and walking/standing separately from the remaining physical demands. All analyses were conducted in SAS V.9.4 using proc mixed for multilevel modelling and applied a level of statistical significance of $\mathrm{p}<0.05$.

\section{RESULTS}

Table 1 shows the characteristics of the participants. There were slightly more women (53\%) than men. The mean age at baseline was 46 years and 45 years for men and women, respectively. The mean level of musculoskeletal pain at baseline was 1.45 for men and 1.65 for women on a scale ranging $0-5$. An overview of the job groups of the participants at the three-digit DISCO level is given in supplementary appendix table A2.

The best performance of the JEMs was seen for physical work demands with more than $50 \%$ variance explained at the job group level and an AUC above 0.80 (supplementary appendix table A3 and A4). The JEM-based measures for psychosocial working conditions showed less consistent performance with ICCs ranging from 0.07 (decision authority in women) to 0.36 (emotional demands in women) and AUCs ranging from 0.56 (job strain in men) to 0.86 (violence in women). Online supplementary appendix table A5 and A6 give an overview of the job groups of the highest and lowest levels of exposure and heterogeneity for the continuous measures.

Table 2 shows the estimated associations between the working conditions and musculoskeletal pain at follow-up. Physical work demands predicted pain whether measured at the individual level or using the JEM. On average, men reported musculoskeletal pain in 0.03 more body regions per point increase in physical work demands. This corresponds to a difference of 1.20 comparing men with the highest level of physical work demands (48) those with the lowest level (8). For women, the difference per point on the scale was 0.02 , corresponding to a difference of 0.80 associated with the full range of the scale. Also, decision authority showed a statistically significant association with musculoskeletal pain using both exposure approaches but only statistically significantly in men. A one point increase of decision authority measured at the individual level was associated with 0.12 less painful body regions, corresponding to a 0.48 difference in the mean number of painful body regions for the full range of the scale. Measured at the JEM level, men with the highest level of decision latitude reported pain in 1.76 more body regions, when compared with men with the lowest JEM measured level of decision latitude.

Violence was consistently associated with musculoskeletal pain in women whether measured at the individual level or JEM level. High job insecurity was associated with more musculoskeletal pain in men when measured with JEM, whereas higher quantitative demands at the JEM level were associated with less pain at follow-up in both men and women.

\section{Sensitivity analysis}

The sensitivity analyses are presented in tables 3 and 4 . Results were largely similar when conducted separately in participants without pain at baseline and participants with one or more pain sites at baseline. The association of higher JEM-level quantitative demands and decision authority with less musculoskeletal pain, however, was more pronounced in participants with some baseline pain than in participants without baseline pain. When distinguishing sitting and walking/standing from the remaining physical work demands, results were similar to those presented 
Table 1 Participant characteristics

\begin{tabular}{|c|c|c|c|c|c|c|c|c|c|c|}
\hline & \multicolumn{5}{|l|}{ Men } & \multicolumn{5}{|c|}{ Women } \\
\hline & N & $\%$ & Mean & SD & Range & N & $\%$ & Mean & SD & Range \\
\hline \multicolumn{11}{|l|}{ Demographics and musculoskeletal pain } \\
\hline Age & & & 46.2 & 10.6 & $18-64$ & & & 45.5 & 10.1 & $18-64$ \\
\hline \multicolumn{11}{|l|}{ Education } \\
\hline Basic school 8-10 grade & 469 & 12.3 & & & & 393 & 9.1 & & & \\
\hline Vocational education & 1454 & 38.2 & & & & 1394 & 32.2 & & & \\
\hline Short-cycle higher education & 294 & 7.7 & & & & 268 & 9.5 & & & \\
\hline Medium-cycle higher education & 646 & 17.0 & & & & 1315 & 30.4 & & & \\
\hline Bachelor & 66 & 1.73 & & & & 100 & 2.3 & & & \\
\hline Long-cycle higher education & 542 & 14.2 & & & & 514 & 11.9 & & & \\
\hline Musculoskeletal pain at follow-up & & & 1.39 & 1.41 & $0-5$ & & & 1.70 & 1.50 & $0-5$ \\
\hline \multicolumn{11}{|l|}{ Continuous exposures } \\
\hline Physical work demands & & & 18.02 & 7.31 & $8-48$ & & & 17.47 & 6.61 & $8-48$ \\
\hline Quantitative demands & & & 3.08 & 0.68 & $1-5$ & & & 2.93 & 0.68 & $1-5$ \\
\hline Emotional demands & & & 2.76 & 1.02 & $1-5$ & & & 3.27 & 1.04 & $1-5$ \\
\hline Decision authority & & & 4.20 & 0.76 & $1-5$ & & & 4.21 & 0.72 & $1-5$ \\
\hline \multicolumn{11}{|l|}{ Dichotomous exposures } \\
\hline High physical work demands (highest decile) & 345 & 9.1 & & & & 374 & 8.6 & & & \\
\hline High quantitative demands (highest decile) & 438 & 11.5 & & & & 603 & 13.9 & & & \\
\hline High emotional demands (highest decile) & 282 & 7.4 & & & & 192 & 4.4 & & & \\
\hline Low decision authority (lowest decile) & 187 & 4.9 & & & & 160 & 3.7 & & & \\
\hline
\end{tabular}

for the complete sum score for physical work demands (results available on request).

\section{DISCUSSION}

Overall, we found predominately similar associations of physical and psychosocial working conditions with musculoskeletal pain, whether measuring working conditions at the JEM or individual level. Generally, any associations that were consistent at the individual level were also found in the JEM-level analysis. We saw consistent associations with musculoskeletal pain for physical work demands, decision authority in men and violence in women, with both exposure approaches. We also found consistent absence of associations in both approaches for emotional demands measured dichotomously, job strain, job insecurity in women and violence in men. There were also several differences across exposure approaches though, most notably, the JEM-level association of higher quantitative demands with less pain, not present at the individual level. This finding suggests the importance of interpreting analyses of job group level data at the job group level, as they cannot necessarily be extrapolated to the individual level.

Of the examined working conditions, we found the most consistent associations with pain for physical work demands. This finding is in line with previous prospective studies reporting associations between self-reported physical work demands and multisite musculoskeletal pain. ${ }^{12} 29$ A suggested mechanism is that high physical work demands lead to increased levels of inflammation, which can modify the autonomous nervous system regulation and/or pain perception, causing multisite pain. ${ }^{30}$ However, both the causality and potential mechanisms behind physical work demands and multisite pain remain much debated.

Five previous validation studies of JEMs constructed by aggregating self-reported data have compared risk estimates using individual-level measurements and JEMs in relation to either low back pain, ${ }^{34}$ mental health ${ }^{46}$ or self-rated health. ${ }^{5}$ All studies analysed the data cross-sectionally and applied binary definitions of the examined exposures. Generally, the studies found weaker associations with health outcomes in JEM-level analyses than using individual-level measures, which is also expected given the exposure misclassification inherent in the JEM approach. ${ }^{31}$ In our study, the JEM-level measurements did not generally lead to lower estimates than the individual-level measurements. One explanation could be that we used a model-based approach to construct the JEM, whereas previous studies used means or prevalences of exposure within job groups. Theoretically, the model-based JEMs should yield unbiased associations because measurement error, under certain assumptions, would predominately be Berkson error, resulting in increased statistical uncertainty of the estimates but non-biased associations. ${ }^{32}$ Another explanation might be that the previous studies compared cross-sectional associations of individual-level and JEM-level exposures with outcomes. At the individual level, such exposure data might be 
Table 2 The effect on musculoskeletal pain at follow-up from working conditions measured at the individual level or job exposure matrix (JEM) level

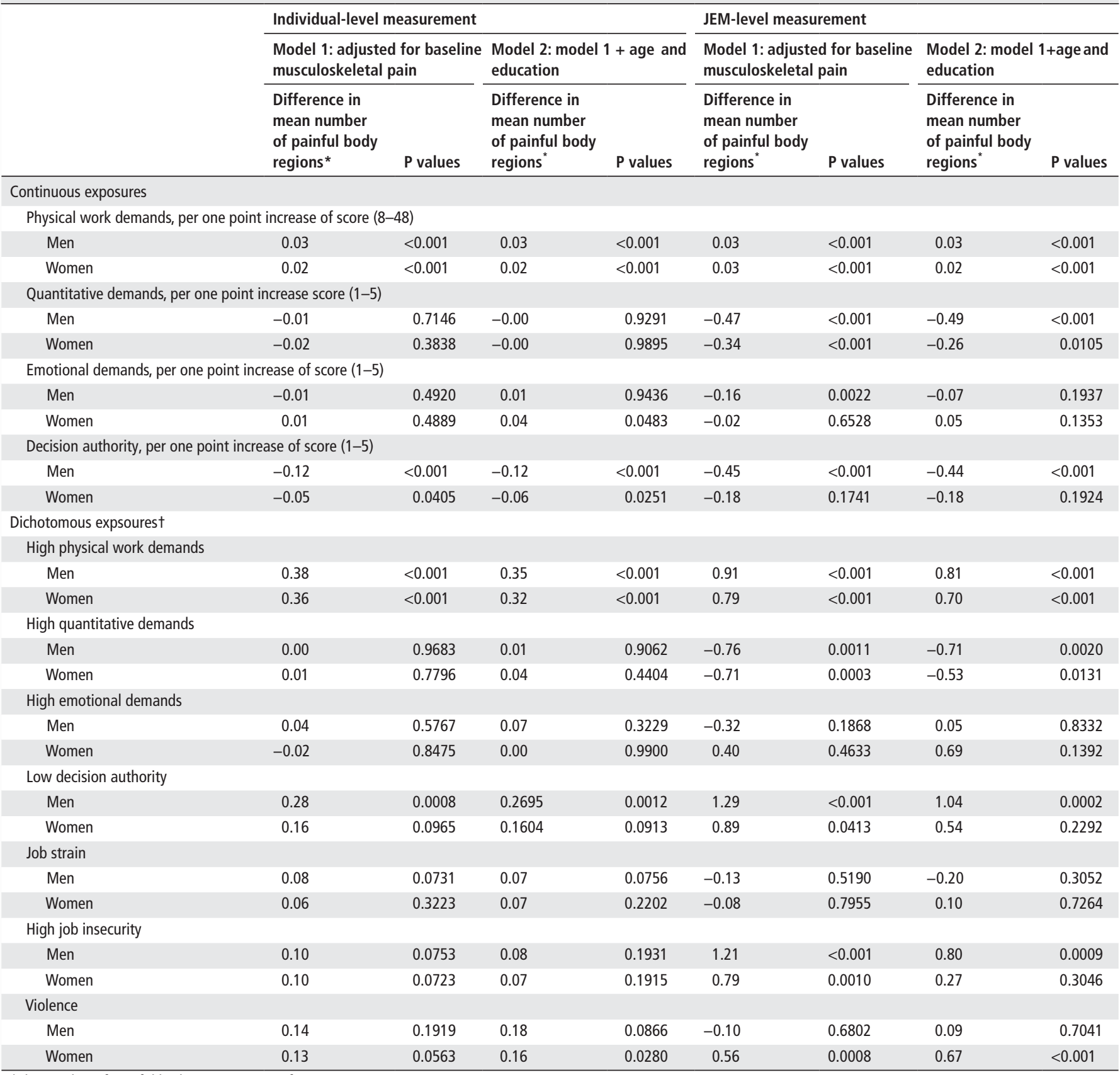

*The number of painful body regions ranges from 0 to 5.

†Association estimates for dichotomous exposures compare high versus low (violence and job strain: yes/no) in individual-level analyses and the risk associated with a $1 \%$ increased risk of exposure in JEM-level analyses.

affected by reporting bias ${ }^{33}$ leading to inflated risk estimates. Using a longitudinal approach, the results from the individual-level exposure data in our study are less prone to such bias, and inference may more readily be drawn, regarding the comparability of individual-level data and JEMs for longitudinal analyses on working conditions and health.

Findings from previous studies on JEM-level psychosocial working conditions have been most consistent for low decision latitude, which has been related to poorer mental health, ${ }^{6}$ self-reported health ${ }^{5}$ and higher risk of anxiety disorders in men. ${ }^{8}$ One study examined prevalence of low back pain as the outcome ${ }^{4}$ and found associations with JEM-level monotonous work. Also, after accounting for exposure misclassification by the JEM, the study found higher risk of low back pain in participants with low decision latitude, low social support, high job strain and high demands in men. We found that higher decision authority, a component of decision latitude, was associated with less pain, although only statistically significantly in men. However, we also found that higher JEM-level quantitative demands were associated with less pain at follow-up. This finding is puzzling, particularly given the opposite direction of the association between physical work demands and musculoskeletal pain. The measurement of quantitative demands included items on the boundarylessness of work, which might better capture demands in job groups of higher socioeconomic position, than demands in job groups of lower socioeconomic 
Table 3 The effect on musculoskeletal pain at follow-up from working conditions in participants with no pain at baseline

\begin{tabular}{|c|c|c|c|c|}
\hline & \multicolumn{2}{|c|}{ Individual-level measurement } & \multicolumn{2}{|c|}{ Job exposure matrix (JEM) } \\
\hline & $\begin{array}{l}\text { Difference in mean } \\
\text { number of painful } \\
\text { body regions* }\end{array}$ & $P$ values & $\begin{array}{l}\text { Difference in mean } \\
\text { number of painful } \\
\text { body regions }\end{array}$ & $P$ values \\
\hline \multicolumn{5}{|c|}{ Continuous exposures } \\
\hline \multicolumn{5}{|c|}{ Physical work demands, per one point increase of score (8-48) } \\
\hline Men & 0.02 & $<0.001$ & 0.02 & 0.0111 \\
\hline Women & 0.03 & $<0.001$ & 0.02 & 0.0010 \\
\hline \multicolumn{5}{|c|}{ Quantitative demands, per one point increase score $(1-5)$} \\
\hline Men & 0.01 & 0.7141 & -0.23 & 0.1319 \\
\hline Women & 0.04 & 0.3241 & -0.18 & 0.2400 \\
\hline \multicolumn{5}{|c|}{ Emotional demands, per one point increase of score (1-5) } \\
\hline Men & 0.00 & 0.9030 & -0.03 & 0.6799 \\
\hline Women & 0.06 & 0.0494 & 0.05 & 0.3914 \\
\hline \multicolumn{5}{|c|}{ Decision authority, per one point increase of score (1-5) } \\
\hline Men & -0.12 & 0.0008 & -0.27 & 0.0780 \\
\hline Women & -0.01 & 0.8668 & -0.00 & 0.9881 \\
\hline \multicolumn{5}{|c|}{ Dichotomous expsoures ${ }^{\dagger}$} \\
\hline \multicolumn{5}{|c|}{ Job strain } \\
\hline Men & 0.03 & 0.5588 & -0.07 & 0.8017 \\
\hline Women & 0.02 & 0.8386 & 0.03 & 0.9402 \\
\hline \multicolumn{5}{|c|}{ High job insecurity } \\
\hline Men & 0.13 & 0.1729 & 0.19 & 0.5957 \\
\hline Women & 0.11 & 0.2685 & 0.21 & 0.6230 \\
\hline \multicolumn{5}{|l|}{ Violence } \\
\hline Men & 0.09 & 0.5899 & 0.66 & 0.0598 \\
\hline Women & 0.16 & 0.0419 & 0.43 & 0.1060 \\
\hline
\end{tabular}

${ }^{*}$ Adjusted for baseline age and education. The number of painful body regions ranges from 0 to 5 .

tAssociation estimates for dichotomous exposures compare high versus low (violence and job strain: yes/no) in individual-level analyses and the risk associated with a $1 \%$ increased risk of exposure in JEM-level analyses.

position, whereas physical work demands are more prevalent in lower socioeconomic position jobs. ${ }^{34}$ The distribution of the JEM-level measurements of the two exposures also showed the highest levels of physical work demands in manual occupations and the highest levels of quantitative demands in managerial occupations (online supplementary appendix table A5). Although we adjusted for a detailed measure of baseline education, we cannot rule out residual confounding by socioeconomic position, and more research to disentangle effects of working conditions and socioeconomic position seems warranted.

For the continuous measures of working conditions, we constructed dichotomised versions, classifying the $10 \%$ most exposed participants as highly exposed. We conducted this dichotomisation to allow for the possibility that the JEM might better capture the percentage of highly exposed participants within job groups than distinguish mean differences between groups. The JEMs performed rather similarly, though, when comparing the two versions. If there was a low ICC for the continuous measure, there was usually also a low AUC for the dichotomised measure. One exception was decision authority, with low ICCs but relatively high AUCs. The associations of decision authority with pain were similar for the continuous and the dichotomised measures of the construct, possibly calling into question the usefulness of the ICC as a quality indicator for JEMs. Further research is needed to establish
Table 4 The effect on musculoskeletal pain at follow-up from working conditions in participants with one or more pain sites at baseline

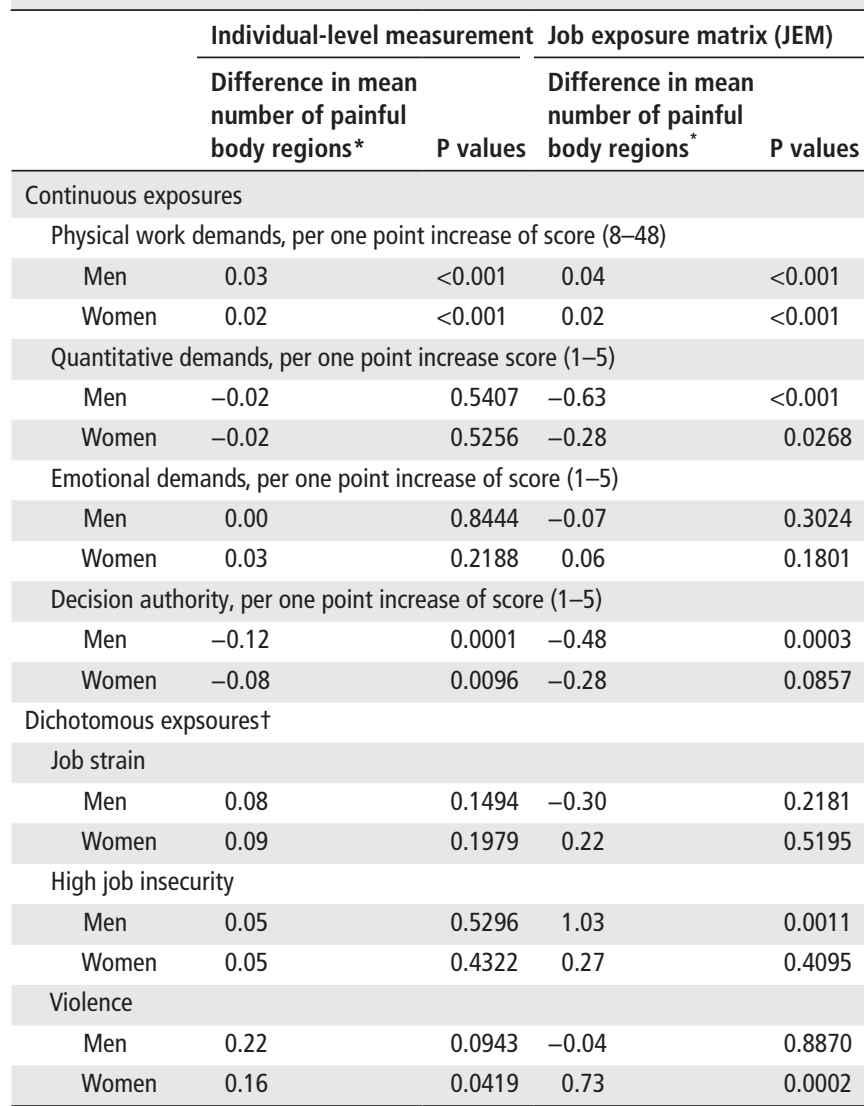

*Adjusted for baseline musculoskeletal pain, age and education. The number of painful body regions ranges from 0 to 5 .

tAssociation estimates for dichotomous exposures compare high versus low (violence and job strain: yes/no) in individual-level analyses and the risk associated with a $1 \%$ increased risk of exposure in JEM-level analyses.

better performance indicators for continuously measured JEMs, possibly incorporating both the variance explained by job group and the exposure contrast.

\section{Strengths and limitations}

The strengths of this study include the model-based approach to estimating JEMs that are sex-specific and age-specific, the longitudinal design and the study population based on a random sample of the Danish working population, strengthening the generalisability of results. Some limitations should, however, be noted. First, the response rate to the WEHD study was $50 \%$, with respondents more likely than non-respondents to be women, older and have longer education. ${ }^{35}$ It is possible that the findings of the present study, despite the random sample, are less generalisable to younger men with shorter education. Second, a relatively high proportion of respondents were excluded due to missing data on pain at baseline $(13 \%)$ or follow-up (10\%). This proportion might be related to the item formulation, where participants were asked to answer yes or no to eight pain sites. Individuals with pain in only few bodily regions may have answered yes to those regions but left the remaining boxes blank, and for these individuals, a sum score could not be calculated. Third, we included no information on several factors that might influence pain, including body mass index, ${ }^{36}$ and mental distress. ${ }^{37}$ We focused mainly on 
comparing the results using JEMs to those using self-reported individual-level measures, and thus included the most essential covariates available in register-based studies. However, the associations could be regarded as minimally adjusted, and caution is warranted in interpreting them as causal. Fourth, the three scales on psychosocial working conditions were specifically constructed for WEHD without formal scale validation. Although the items of the scales were derived and modified from previously validated Danish questionnaires, ${ }^{38-40}$ the lack of formal validation of the specific scales remains a limitation of this study.

\section{CONCLUSION}

To conclude, we found predominately consistent prospective associations between working conditions and pain, whether using JEMs or individual-level exposures. The results suggest that, with a few notable exceptions, particularly quantitative demands, most findings obtained using JEMs may be comparable with those obtained when measuring physical work and psychosocial working conditions by self-report.

Correction notice This article has been corrected since it was first published. It has been made open access.

Contributors IEHM, NG, EB-J, JPB, EF, EMF, SBP, AH and RR contributed to the conception and design of the study, and all authors contributed to the interpretation of the data. IEHM conducted the data analysis and drafted the manuscript, and all authors critically revised it for important intellectual content and approved the final version. All authors agree to be accountable for all aspects of the work in ensuring that questions related to the accuracy or integrity of any part of the work are appropriately investigated and resolved.

Funding This work was funded by the Danish Work Environment Research Fund, grant numbers 43-2014-03 and 17-2014-03, and Nordforsk grant number 75021.

Competing interests None declared.

Patient consent Not required.

Provenance and peer review Not commissioned; externally peer reviewed.

Open access This is an open access article distributed in accordance with the Creative Commons Attribution Non Commercial (CC BY-NC 4.0) license, which permits others to distribute, remix, adapt, build upon this work non-commercially, and license their derivative works on different terms, provided the original work is properly cited, appropriate credit is given, any changes made indicated, and the use is non-commercial. See: http://creativecommons.org/licenses/by-nc/4.0/.

\section{REFERENCES}

1 Coggon D, Pannett B, Acheson ED. Use of job-exposure matrix in an occupational analysis of lung and bladder cancers on the basis of death certificates. J Nat/ Cancer Inst 1984:72:61-5.

2 Hinds MW, Kolonel LN, Lee J. Application of a job-exposure matrix to a case-control study of lung cancer. J Natl Cancer Inst 1985;75:193-7.

3 Solovieva S, Pehkonen I, Kausto J, et al. Development and validation of a job exposure matrix for physical risk factors in low back pain. PLoS One 2012;7:e48680.

4 Solovieva S, Pensola T, Kausto J, et al. Evaluation of the validity of job exposure matrix for psychosocial factors at work. PLoS One 2014;9:e108987.

5 Niedhammer I, Milner A, LaMontagne AD, et al. Study of the validity of a job-exposure matrix for the job strain model factors: an update and a study of changes over time. Int Arch Occup Environ Health 2018;91:523-36.

6 Milner A, Niedhammer I, Chastang JF, et al. Validity of a job-exposure matrix for psychosocial job stressors: results from the household income and labour dynamics in Australia Survey. PLoS One 2016;11:e0152980.

7 Sundstrup E, Hansen ÅM, Mortensen EL, et al. Cumulative occupational mechanica exposures during working life and risk of sickness absence and disability pension: prospective cohort study. Scand J Work Environ Health 2017:43:415-25.

8 Wieclaw J, Agerbo E, Mortensen PB, et al. Psychosocial working conditions and the risk of depression and anxiety disorders in the Danish workforce. BMC Public Health 2008;8:280.

9 Podsakoff PM, MacKenzie SB, Lee JY, Jeong-Yeon L, et al. Common method biases in behavioral research: a critical review of the literature and recommended remedies. $J$ Appl Psychol 2003;88:879-903.

10 GDallaP C. GBD 2016 Disease and Injury Incidence and Prevalence Collaborators. Global, regional, and national incidence, prevalence, and years lived with disability for
328 diseases and injuries for 195 countries, 1990-2016: a systematic analysis for the Global Burden of Disease Study 2016. Lancet 2017:390:1211-59.

11 Hauke A, Flintrop J, Brun E, et al. The impact of work-related psychosocial stressors on the onset of musculoskeletal disorders in specific body regions: A review and metaanalysis of 54 longitudinal studies. Work Stress 2011;25:243-56.

12 Neupane S, Leino-Arjas P, Nygård CH, et al. Developmental pathways of multisite musculoskeletal pain: what is the influence of physical and psychosocial working conditions? Occup Environ Med 2017;74:468-75.

13 Oakman J, de Wind A, van den Heuvel SG, et al. Work characteristics predict the development of multi-site musculoskeletal pain. Int Arch Occup Environ Health 2017:90:653-61.

14 Wai EK, Roffey DM, Bishop P, et al. Causal assessment of occupational lifting and low back pain: results of a systematic review. Spine J 2010;10:554-66.

15 Roffey DM, Wai EK, Bishop P, et al. Causal assessment of workplace manual handling or assisting patients and low back pain: results of a systematic review. Spine J 2010;10:639-51

16 Koch P, Schablon A, Latza U, et al. Musculoskeletal pain and effort-reward imbalance-a systematic review. BMC Public Health 2014;14:37.

17 Kuorinka I, Jonsson B, Kilbom A, et al. Standardised Nordic questionnaires for the analysis of musculoskeletal symptoms. Appl Ergon 1987;18:233-7.

18 Andersen LL, Fallentin N, Thorsen SV, et al. Physical workload and risk of longterm sickness absence in the general working population and among blue-collar workers: prospective cohort study with register follow-up. Occup Environ Med 2016;73:246-53.

19 Madsen IE, Diderichsen F, Burr H, et al. Person-related work and incident use of antidepressants: relations and mediating factors from the Danish work environment cohort study. Scand I Work Environ Health 2010;36:435-44.

20 Madsen IEH, Nyberg ST, Magnusson Hanson LL, et al. Job strain as a risk factor for clinical depression: systematic review and meta-analysis with additional individual participant data. Psychol Med 2017;47:1342-56.

21 (ILO) ILO. ISCO-08 Structureindex correspondence with ISCO-88: International Labour Organization (ILO), 2016. http://www.ilo.org/public/english/bureau/stat/isco/isco08/ index.htm.

22 Baadsgaard M, Quitzau J. Danish registers on personal income and transfer payments. Scand J Public Health 2011;39:103-5.

23 Hanley JA, McNeil BJ. The meaning and use of the area under a receiver operating characteristic (ROC) curve. Radiology 1982;143:29-36.

24 Hajian-Tilaki K. Receiver operating characteristic (roc) curve analysis for medical diagnostic test evaluation. Caspian J Intern Med 2013;4:627-35.

25 Youngstrom EA. A primer on receiver operating characteristic analysis and diagnostic efficiency statistics for pediatric psychology: we are ready to ROC.J Pediatr Psychol 2014;39:204-21.

26 Zhou X-H, McClish DK, Obuchowski NA P. A Review of: "Statistical Methods in Diagnostic Medicine, 2011.

27 Pedersen CB. The Danish Civil Registration System. Scand J Public Health 2011;39:22-5

28 Jensen VM, Rasmussen AW. Danish education registers. Scand J Public Health 2011:39:91-4

29 Coggon D, Ntani G, Palmer KT, et al. Patterns of multisite pain and associations with risk factors. Pain 2013:154:1769-77.

30 Christian M, Nussbaum MA. An exploratory study of the effects of occupational exposure to physical demands on biomarkers of cartilage and muscle damage. $J$ Occup Environ Hyg 2015;12:138-44.

31 Kauppinen TP. Development of a classification strategy of exposure for industry-based studies. Appl Occup Environ Hyg 1991;6:482-7.

32 Berkson J. Are there Two Regressions? J Am Stat Assoc 1950;45:164-80.

33 Kolstad HA, Hansen AM, Kærgaard A, et al. Job strain and the risk of depression: is reporting biased? Am J Epidemiol 2011;173:94-102.

34 Hämmig O, Bauer GF. The social gradient in work and health: a cross-sectional study exploring the relationship between working conditions and health inequalities. BMC Public Health 2013:13:1170.

35 Nye resultater fra undersøgelsen 'Arbejdsmiljø og Helbred i Danmark 2016' (AH2016, 2013.

36 Heuch I, Heuch I, Hagen K, et al. Body mass index as a risk factor for developing chronic low back pain: a follow-up in the Nord-Trøndelag Health Study. Spine 2013:38:133-9.

37 Christensen JO, Johansen S, Knardahl S. Psychological predictors of change in the number of musculoskeletal pain sites among Norwegian employees: a prospective study. BMC Musculoskelet Disord 2017;18:140.

38 Pejtersen JH, Kristensen TS, Borg V, et al. The second version of the Copenhagen Psychosocial Questionnaire. Scand J Public Health 2010;38:8-24.

39 Bjorner JB, Pejtersen JH. Evaluating construct validity of the second version of the copenhagen psychosocial questionnaire through analysis of differential item functioning and differential item effect. Scand I Public Health 2010;38:90-105.

40 Clausen T, Madsen IEH, Christensen KB, et al. Dansk psykosocialt spørgeskema - et spørgeskema om psykosocialt arbejdsmiljø [Danish Psychosocial Questionnaire - A questionnaire on psychosocial working conditions]. Copenhagen: The National Research Centre for the Working Environment, 2017. 